\title{
Low resolution spectroscopy of hot post-AGB candidates ${ }^{\star}$
}

\author{
M. Parthasarathy ${ }^{1,2}$, J. Vijapurkar ${ }^{3}$, and J.S. Drilling ${ }^{4}$ \\ 1 National Astronomical Observatory, 2-21-1 Osawa, Mitaka, Tokyo 181-8588, Japan \\ 2 Indian Institute of Astrophysics, Koramangla, Bangalore-560034, India \\ 3 Inter-University Center for Astronomy and Astrophysics (IUCAA), Post Bag 4, Ganeshkhind, Pune 411007, India \\ 4 Department of Physics and Astronomy, Louisiana State University, Baton Rouge, Louisiana 70803, U.S.A.
}

Received February 14; accepted May 23, 2000

\begin{abstract}
Spectral types are determined from low resolution optical spectra of 40 IRAS sources with far-infrared colours similar to planetary nebulae. The presence of circumstellar dust, high galactic latitude, and early supergiant or giant spectral type, sometimes with emission in the Balmer lines, indicate that some of these stars are low mass stars in the post-AGB stage of evolution.
\end{abstract}

Key words: stars: AGB and post-AGB - stars: evolution — stars: early-type — stars: emission-line stars: atmospheres

\section{Introduction}

From an analysis of the IRAS point source catalogue data, several post-asymptotic giant branch (post-AGB) candidates were detected (Parthasarathy \& Pottasch 1986; Pottasch \& Parthasarathy 1988; Parthasarathy 1993a; Kwok 1993). The post-AGB stars, i.e. the nuclei of protoplanetary nebulae (PPNe) and planetary nebulae $(\mathrm{PNe})$, were identified on the basis of far-infrared (IRAS) colours. In order to further understand the evolutionary status of the optical counterparts, it is often necessary to carry out multi-wavelength spectroscopy and photometry. The post-AGB stars detected from the IRAS data seem to form an evolutionary sequence $(\mathrm{K}, \mathrm{G}, \mathrm{F}, \mathrm{A}$ to $\mathrm{OB}$ supergiant types) in the transition region from the tip of the AGB to the early stages of planetary nebulae (Parthasarathy 1993a,b, 1994; Parthasarathy et al. 1993). The PPNe and PNe have more or less the same far-IR colours (Parthasarathy \& Pottasch 1986; Pottasch et al. 1988). Spectral classification based on low resolution optical spectra of several IRAS sources was carried out by

Send offprint requests to: J.S. Drilling

* Based on observations obtained at the Cerro Tololo Inter-American Observatory (CTIO), Chile.
Hrivnak et al. (1989). They have found several F and G type post-AGB candidates, but very few hot post-AGB candidates are known. In order to understand the evolution from cooler to hotter post-AGB types and then into the young $\mathrm{PN}$ stage, it is important to detect and study several hot post-AGB candidates. In order to detect hot post-AGB candidates and to determine their spectral characteristics, we have obtained low resolution blue spectra of several IRAS sources with far-IR colours similar to $\mathrm{PNe}$ and PPNe. In this paper we present the results based on spectra of selected IRAS sources obtained with the $1 \mathrm{~m}$ telescope of the Cerro Tololo Inter-American Observatory (CTIO), Chile.

\section{Selection criteria}

The PNe and PPNe form a broad but limited group in the IRAS colour-colour diagram (Pottasch et al. 1988; van der Veen \& Habing 1988). They are reasonably well separated from most of the other objects. Based on the IRAS colourcolour diagram one can conclude that there is a good chance that an object is a PNe or PPNe if its IRAS colours $F(12 \mu \mathrm{m}) / F(25 \mu \mathrm{m})<0.35$ and $F(25 \mu \mathrm{m}) / F(60 \mu \mathrm{m})$ $>$ 0.3. An occasional HII region, Seyfert galaxy, or T-Tau star is not excluded from this range. With the above mentioned criteria, the IRAS point source catalogue was searched to select the post-AGB candidates. We have considered only the sources which have good quality fluxes at 100 microns (a flux quality flag of 2 or 3 in the IRAS point source catalog) and which have optical counterparts brighter than 14th magnitude. From this we made a short list of southern objects (which can be observed from CTIO) which are associated with early type (OB) stars in the HD or SAO catalogues, or with early type emission line stars (Henize 1976; Wackerling 1970). Some of these stars were listed in two earlier papers (Parthasarathy \& Pottasch 1989; Parthasarathy 1993a). We also found that some of the LS, LSS and 
LSE stars (Hardrop et al. 1959; Stephenson \& Sanduleak 1971; Drilling \& Bergeron 1995 and references therein) are IRAS sources with far-IR colours similar to PPNe and PNe (Parthasarathy 1993a,b and 1994; Conlon et al. 1993).

\section{Observations}

Digital spectra of the selected southern candidates were obtained in April 1994 using the spectrograph and 2d-Frutti two-dimensional photon counting detector on the $1 \mathrm{~m}$ telescope of the Cerro Tololo Inter-American Observatory in Chile. The wavelength coverage is $3800 \AA$ to $5000 \AA$, and judging from the comparison spectra, the resolution is about $3.5 \AA$. The data were extracted, wavelength calibrated, and normalized to the continuum with the standard IRAF software.

\section{Analysis}

\subsection{Spectral classification}

Since most of our targets are of OB spectral type, we have compared the spectra of our program stars with the spectra of standard OB stars (Walborn \& Fitzpatrick 1990). Walborn and Fitzpatrick made a digital atlas of the the spectra of OB stars which they observed with the same instrument, but at higher resolution than that described above. We found that smoothing the Walborn and Fitzpatrick spectra by $3.5 \AA$ produced nearly identical looking spectra for the $09.5 \mathrm{~V}$ star HD 37468, which we observed, and the Walborn and Fitzpatrick O9.5V standard HD 93027. Spectral types for non-OB stars were estimated by comparison with the photographic atlas of Yamashita et al. (1978). For most of our program stars, only rough (if any) spectral type information is available in the literature, and this is listed in Col. 4 of Table 1. The spectral types determined from the present investigation are given in Col. 5. The presence of circumstellar dust with far-IR colours similar to $\mathrm{PNe}$, high galactic latitude, OB supergiant type spectrum, and emission in the Balmer lines are some of the characteristics of hot post-AGB stars. Table 1 contains several hot post-AGB candidates. Further confirmation of their post-AGB evolutionary stage requires chemical composition analysis, detection of radio continuum emission, mid-IR imaging, IR spectroscopy to distinguish between carbon-rich and oxygen rich dust, and high resolution imaging with WFPC2 and NICMOS to detect nebulae. In Table 1 we have a few objects which are not post-AGB stars. They were included in the observing program as some of them have IRAS colours overlapping with the IRAS colours of some known post-AGB stars. In the next section we give notes on some of the objects.

\subsection{Notes on individual objects in Table 1}

$$
\text { \# IRAS 09470-4617 (= LSS 1351) }
$$

We find the CIII lines in our spectrum of LSS 1351 to be stronger than those of a standard star of similar spectral type. The Balmer lines are filled in. The high galactic latitude, circumstellar dust, B1Iae spectral type and strength of the CIII lines suggest that it may be a carbon-rich postAGB star. Chemical composition determination from an analysis of high resolution spectra may enable us to further understand the evolutionary state of this star.

\section{\# IRAS 10178-5958 (= Hen 401)}

Henize (1976) identified it as an early type emission line star. Parthasarathy \& Pottasch (1989) found it to be an IRAS source with far-IR colours similar to planetary nebulae. They suggested that it is a hot post-AGB star. It is a bipolar reflection nebula surrounding a reddened B1e star (Sanduleak \& Stephenson 1973; Allen 1978). Bujarrabal \& Bachiller (1991) and Loup et al. (1990) found CO line emission at 2.6 and $1.3 \mathrm{~mm}$, implying the presence of a dense extended molecular envelope expanding at $15 \mathrm{~km} \mathrm{~s}^{-1}$. Recently Sahai et al. (1999) obtained high resolution images of Hen 401 with the Wide Field Planetary Camera 2 (WFPC2) on board the Hubble Space Telescope (HST). The HST images of Hen 401 revealed two very long (each $14.5^{\prime \prime}$ ) cylindrical-shaped bipolar outflow lobes, each with a length/width ratio of about 7 , probably the largest seen in proto-planetary nebulae. Recently, GarciaLario et al. (1999) also found similar results based on spectroscopy and near infrared imaging with HST. Our optical spectrum of Hen 401 shows the Balmer lines in emission. The [SII] lines at $4070 \AA$ are in emission. Several permitted and forbidden lines of FeII are in emission. Garcia-Lario et al. (1999) have listed the emission lines seen in the optical spectrum of Hen 401. Comparison of the strengths of the emission lines in our spectrum and those of GarciaLario et al. (1999) and Allen (1978) indicates that there have been no significant variations in the positions and strengths of emission lines in the spectrum of Hen 401.

\# IRAS 11065-6026 (= Hen 591)

Henize (1976) identified it as an early-type emission line star. Parthasarathy \& Pottasch (1989) found it to be an IRAS source with far infrared colours similar to planetary nebulae. They suggested that it is a hot post-AGB star. Garcia-Lario et al. (1998) suggest that it is a Luminous Blue Variable (LBV). The Balmer lines are in emission. Several permitted and forbidden lines of FeII are in emission. The emission lines seen in the optical spectrum of Hen 591 are listed by Garcia-Lario et al. (1998). High resolution spectroscopy and photometric monitoring is needed to find out if it is an LBV or a post-AGB star of high core mass. 
Table 1. Spectral types of optical counterparts of IRAS sources

\begin{tabular}{|c|c|c|c|c|c|c|}
\hline IRAS & $b$ & Name & Old Sp. Type & New Sp. Type & mag & comment \\
\hline $07377-2523$ & -1.61 & & & B8III-Ve & 12.5 & \\
\hline 09470-4617 & +5.59 & LSS 1351 & $\mathrm{OB}+\mathrm{r}$ & B1Iae & 12.4 & post-AGB \\
\hline $10178-5958$ & -2.72 & Hen 401 & $\mathrm{Be}$ & $\mathrm{PN}$ & 12.5 & bipolar PPN \\
\hline $11065-6026$ & -0.31 & Hen 591 & $\mathrm{Be}$ & LBV? & 11.8 & post-AGB? \\
\hline $12175-5338$ & +8.67 & -535072 & A9Iab & A7I & 9.4 & post-AGB \\
\hline $13266-5551$ & +6.36 & -555588 & $\mathrm{Be}$ & B1Ibe & 10.7 & post-AGB \\
\hline $14072-5446$ & +6.12 & -545573 & & A3I & 10.9 & post-AGB \\
\hline $14331-6435$ & -4.20 & Hen 1013 & $\mathrm{Be}$ & B3Ie & 10.9 & post-AGB \\
\hline $15141-6238$ & -4.58 & LSS 3332 & $\mathrm{OB}+$ & O8III & 11.3 & \\
\hline $16024-1813$ & +24.60 & & & G8V & 13.5 & $\beta \mathrm{Pic}$ \\
\hline $16206-5956$ & -7.49 & SAO 243756 & B8Ia & A3Ie & 9.7 & post-AGB \\
\hline $16336-5536$ & -5.77 & HD 149427 & & $\mathrm{PN}$ & 11.8 & \\
\hline $16590-5351$ & -7.46 & -538315 & & $\mathrm{PN}$ & 10.5 & \\
\hline $17041-2709$ & +8.00 & Hen 1336 & $\mathrm{Be}$ & $\mathrm{K} 7 \mathrm{~V}$ & 12.2 & T-Tau \\
\hline $17074-1845$ & +12.28 & Hen 1347 & $\mathrm{Be}$ & B3IIIe & 9.5 & post-AGB \\
\hline $17086-2403$ & +8.99 & & & G5IV-V & 11.9 & $\beta \mathrm{Pic}$ \\
\hline $17203-1534$ & +11.49 & & & B1IIIpe & 12.5 & post-AGB \\
\hline $17311-4924$ & -9.04 & Hen 1428 & $\mathrm{Be}$ & B1IIe & 10.7 & post-AGB \\
\hline 17381-1616 & +7.50 & LSS 4331 & $\mathrm{OB}+$ & B1Ibe & 12.2 & post-AGB \\
\hline $17395-0841$ & +11.10 & & & $\mathrm{PN}$ & 13.2 & \\
\hline $17412-0614$ & +11.95 & SAO 141834 & Ape & Ape & 8.9 & \\
\hline $17423-1755$ & +5.78 & Hen 1475 & $\mathrm{Be}$ & $\mathrm{PN}$ & 11.0 & bipolar PPN \\
\hline $17460-3114$ & -1.88 & SAO 209306 & B0.5II & O8III & 7.9 & post-AGB \\
\hline $18023-3409$ & -6.31 & LSS 4634 & $\mathrm{OB}+$ & B2IIIe & 11.6 & post-AGB? \\
\hline $18040-1457$ & +2.78 & & & $\mathrm{~B} 0 \mathrm{~V}$ & 12.5 & \\
\hline $18062+2410$ & +19.79 & SAO 85766 & & B1IIIpe & 11.5 & post-AGB \\
\hline $18070-2346$ & -2.16 & SAO 186389 & $\mathrm{~B} 2 \mathrm{~V}$ & $\mathrm{~B} 2 \mathrm{Vn}$ & 8.0 & \\
\hline $18227-1243$ & -0.13 & RY Scuti & & B1IIIpe & 9.1 & binary \\
\hline $18237-0715$ & +2.23 & MWC 930 & $\mathrm{Be}$ & $\mathrm{Be}$ & 11.0 & \\
\hline $18313-1738$ & -4.27 & MWC 939 & $\mathrm{Be}$ & B5? & 12.4 & \\
\hline $18367-1233$ & -3.09 & LSIV -1297 & OB & B3V & 10.4 & \\
\hline 18371-3159 & -11.82 & LSE 63 & $\mathrm{OB}+$ & B1Iabe & 11.9 & post-AGB \\
\hline 18379-1707 & -5.42 & LSS 5112 & $\mathrm{OB}+\mathrm{h}$ & B1IIIpe & 11.8 & post-AGB \\
\hline $18435-0052$ & +0.81 & & & B2II & 12.5 & post-AGB? \\
\hline $18442-1144$ & -4.34 & $\mathrm{BD}-114747$ & & $\mathrm{~A} 3 \mathrm{~V}$ & 9.3 & see notes \\
\hline $18489-3703$ & -16.09 & & & $\mathrm{~K} 7 \mathrm{~V}$ & 11.9 & T-Tau \\
\hline $19157-0247$ & -7.22 & LSIV -2 29 & B9Ib & B1III & 11.5 & post-AGB \\
\hline 19336-0400 & -11.75 & & & B1Iape & 12.5 & post-AGB \\
\hline $19399+2312$ & +0.08 & LSII + 2317 & $\mathrm{OB}(\mathrm{ce}) \mathrm{h}, \mathrm{r}$ & B1III & 10.4 & post-AGB? \\
\hline 19590-1249 & -21.26 & LSIV -12 111 & $\mathrm{OB}+\mathrm{h}$ & B1Ibe & 11.3 & post-AGB \\
\hline
\end{tabular}

\# IRAS 12175-5338 (= SAO 239853 = LSS 2646)

On the basis of IRAS colours, high galactic latitude and A supergiant spectral class, Parthasarathy (1993a) classified it as a post-AGB star.

\section{\# IRAS 13266-5551 (= -55 5588 = LSS 3099)}

Based on IRAS colours and high galactic latitude, Parthasarathy (1993a) classified it as a post-AGB star. We find the spectral type to be B1Ibe. The Balmer lines are in emission up to $\mathrm{H} \delta$.
\# IRAS 14072-5446 (= CPD -54 5573)

High galactic latitude, A3 supergiant spectral type, detached cold circumstellar dust and $\mathrm{H} \alpha$ emission suggests that CPD -54 5573 is a post-AGB star.

\# IRAS 14331-6435 (= Hen 1013 = LSS 3268)

Henize (1976) identified it as an $\mathrm{H} \alpha$ emission line star. Parthasarathy \& Pottasch (1989) found it to be an IRAS source with far-IR colours similar to PNe and suggested that it is in the post-AGB stage of evolution. We find it to be a $\mathrm{B} 3$ supergiant with $\mathrm{H} \beta$ in emission and $\mathrm{H} \gamma$ filled in. 


\section{\# IRAS 15141-6238}

Its optical counterpart is LSS 3332. High galactic latitude, O8III spectrum and the presence of circumstellar dust indicates that it is a post-AGB star. However high resolution spectroscopy may enable us to further understand its evolutionary status.

\section{\# IRAS 16024-1813}

It is a high galactic latitude star $(b=+24.6)$. Our spectrum shows that it is a G8 dwarf with the $\mathrm{H}$ and $\mathrm{K}$ lines of Ca II in emission. It may be a Vega excess or $\beta$ Pic type star.

\# IRAS 16206-5956 (= SAO 243756)

On the basis of IRAS data, high galactic latitude and supergiant type spectrum, Parthasarathy (1993a) considered it to be a post-AGB star. We find the spectral type to be A3Ie. $\mathrm{H} \beta$ is in emission.

$$
\text { \# IRAS 16336-5536 (= HD } 149427 \text { = PC 11) }
$$

Allen (1982) considered it to be a D' symbiotic star. Recent detailed analysis of the optical spectrum by Gutierrez-Moreno \& Moreno (1998) shows that it is a PN. We also classify the spectrum as that of a PN.

$$
\text { \# IRAS 16590-5351 (= -53 8315) (PN G 334.8-07.4) }
$$

We classify the spectrum as that of a PN. Acker et al. (1992) also classify the spectrum as that of a PN. The Ca II K line at $3933 \AA$ is found to be in absorption. It is strong and broad, indicating that it may not be of interstellar origin. The presence of the Ca II absorption line suggests a late type binary companion to the PN central star. Another possibility is that this PN has a significant amount of neutral gaseous envelope in addition to circumstellar dust.

$$
\text { \# IRAS 17041-2709 (= Hen 1336) }
$$

Parthasarathy \& Pottasch (1989) considered it to be a post-AGB star on the basis of far-infrared fluxes and colours. Henize (1976) found it to show $\mathrm{H} \alpha$ in emission and considered it to be a Be star. Kozok (1985a,b) made $U B V$ observations and derives $V=12.17, B-V=1.13$. We find the spectral type to be K7V. The spectrum is very similar to that of IRAS 18489-3703. The Balmer lines and $\mathrm{H}$ and $\mathrm{K}$ lines of $\mathrm{Ca}$ II are in emission. Presence of circumstellar dust and the optical spectrum suggest that it is likely a weak line T-Tau star. A plot of the spectrum is given by Vijapurkar et al. (1998).

\# IRAS 17074-1845 (= Hen 1347 = BD -18 4436)

On the basis of high galactic latitude, far-infrared colours similar to PNe and Be spectral type (Henize 1976), Parthasarathy (1993a) classified it as a hot post-AGB star. Garcia-Lario et al. (1997) made near-IR observations and they also classified it as a post-AGB star. We find the spectral type to be B3IIIe. $\mathrm{H} \beta$ and $\mathrm{H} \gamma$ are in emission.
\# IRAS 17086-2403

We find the spectral type to be G5IV-V. It may be a Vega excess or $\beta$ Pic type star.

\section{\# IRAS 17203-1534}

We find it to be a high galactic latitude B1IIIpe star. $\mathrm{H} \beta$ is in emission. The high galactic latitude, early spectral type, low gravity, Balmer line emission and far-IR colours similar to PNe indicate that it is a post-AGB star.

\# IRAS 17311-4924 (= Hen 1428)

Parthasarathy \& Pottasch (1989), on the basis of IRAS data, classified it as a post-AGB star. High galactic latitude, far-IR colours similar to $\mathrm{PNe}$ and B1IIe spectral type indicate that it is most likely a post-AGB star.

\# IRAS 17381-1616 (= LSS 4331)

It is a high latitude star. We find the spectral type to be B1Ibe. We find the $\mathrm{H} \beta$ and $\mathrm{H} \gamma$ lines in emission. It is most likely a post-AGB star similar to LS II +34 26 (Parthasarathy 1993b).

\section{\# IRAS 17395-0841}

A new low excitation PN discovered from our survey (Vijapurkar et al. 1997).

\section{\# IRAS 17412-0614 (= SAO $141834=$ XX Oph)}

It is a known Ape P-Cygni type star (see De Winter \& The 1990).

\# IRAS 17423-1755 (= Hen 1475)

Henize (1976) identified it as an $\mathrm{H} \alpha$ emission object. Parthasarathy \& Pottasch (1989) found it to be an IRAS source and on the basis of IRAS colours they classified it as a post-AGB star. Bobrowsky et al. (1995) and Riera et al. (1995) found it to be a bipolar planetary nebula. Recently, Borkowski et al. (1997) and Bobrowsky et al. (1995) imaged Hen 1475 in the [NII] $6584 \AA$ line with the Wide Field Planetary Camera on board HST. They find large-scale flows being collimated into narrow bipolar jets. Emission lines of HeI, FeII, [FeII], OI, CaII and [CaII] are detected in the optical and near-IR spectrum (Riera et al. 1995). We also find several emission lines which are in agreement with the list of Riera et al. (1995). We find the Balmer lines to have P-Cygni profiles which are violet shifted by $-400 \mathrm{~km} \mathrm{~s}^{-1}$, indicating a stellar wind and mass loss from the central star.

\section{\# IRAS 17460-3114}

On the basis of IRAS colours and fluxes and B supergiant spectral type, Parthasarathy (1993a) classified it as a hot post-AGB star.

\# IRAS 18023-3409 (= LSS 4634)

It was classified as $\mathrm{OB}+$ in the LSS catalogue. We find the spectral type to be B2IIIe. We find $\mathrm{H} \beta$ in emission 
and $\mathrm{H} \gamma$ appears to be filled in. High galactic latitude, farIR colours similar to $\mathrm{PNe}$ and the B2IIIe spectral type suggest that it is most likely a post-AGB star.

\section{\# IRAS 18062+2410 (SAO 85766)}

It is a high galactic latitude star $(b=+20)$. Based on IRAS colours and high galactic latitude, it was classified as a post-AGB star (Volk \& Kwok 1989; Parthasarathy 1993a). According to the HDE catalogue its spectral type in 1940 was A5. Downes \& Keyes (1988) obtained the spectral energy distribution and classified it as a Be star. Drilling \& Bergeron (1995) in their extension of the CaseHamburg OB-star Surveys listed it as LSE 162 and classified it as $\mathrm{OB}+$. From the spectrum obtained in this survey we find the spectral type to be B1IIIpe. Recently Arkhipova et al. (1999) and Parthasarathy et al. (1999) analysed the high resolution spectrum of this star and find the effective temperature to be $22000 \mathrm{~K}$. They have found numerous permitted and forbidden emission lines of several elements in the high resolution spectrum of this star. The presence of $[\mathrm{NII}]$ and $[\mathrm{SII}]$ lines shows the presence of a low excitation planetary nebula. This star is rapidly evolving into a young planetary nebula.

\section{\# IRAS 18237-0715 (= MWC 930)}

The Balmer lines and several permitted and forbidden lines of $[\mathrm{FeII}]$ are in emission. A plot of the spectrum with line identification is given by Vijapurkar et al. (1998). The IRAS data indicate the presence of a detached cold dust shell. The evolutionary stage of this star is not clear; it may be related to Pop. I Be stars or may be an LBV.

$$
\text { \# IRAS 18313-1738 ( = MWC 939) }
$$

The spectrum of this star is to some extent similar to that of MWC 930. The Balmer lines and several permitted and forbidden lines of Fe are in emission. A plot of the spectrum with line identification is given by Vijapurkar et al. (1998). It may be a Pop. I Be star.

\section{\# IRAS 18371-3159 ( = LSE 63)}

It is classified as $\mathrm{OB}+$ in the extension of the CaseHamburg OB-star surveys (Drilling \& Bergeron 1995). The IRAS colours are similar to $\mathrm{PNe}$. The $\mathrm{H} \beta$ and $\mathrm{H} \gamma$ lines are in emission. We find the spectral type of the star to be B1Iabe. High galactic latitude, IRAS colours similar to $\mathrm{PNe}$, Balmer line emission and $\mathrm{B}$ supergiant spectral type suggest that it is most likely a post-AGB star. It appears to be similar to the post-AGB star LS II +3426 (Parthasarathy 1993b).

$$
\text { \# IRAS 18379-1707 (= LSS 5112) }
$$

We have selected it as a post-AGB candidate on the basis of IRAS colours. Our spectrum shows $\mathrm{H} \beta$ partially filled in and an HeI emission reversal. The object may be metalpoor. We find the spectral type to be B1IIIpe. Venn et al. (1998) find it to be B2.5Ia. They also find $\mathrm{H} \delta$ to be filled in or to have a weak P-Cygni type profile. We conclude that it is most likely a post-AGB star.

\section{\# IRAS 18442-1144}

This source is very close to BD -114747 . We have observed BD -11 4747, as the optical counterpart to IRAS $18442-1144$ is relatively faint. We classify BD -114747 as an A3 V star. IRAS 18442-1144 shows [NII] lines (Henize 1976), and it may be a hot post-AGB star or a low excitation PN. IRAS 18442-1144 and BD -11 4747 may be a visual binary system.

\section{\# IRAS 18489-3703}

A plot of the spectrum is given by Vijapurkar et al. (1998). All Balmer lines are in emission. The $\mathrm{H}$ and $\mathrm{K}$ lines of CaII are also in emission. We find the spectral type to be K7V. The presence of circumstellar dust and the optical spectrum suggest that it may be a weak line T-Tau star. The spectrum of this star is similar to that of IRAS 170412709 .

\section{\# IRAS 19336-0400}

A low resolution and low signal-to-noise spectrum of this star was published by Downes \& Keyes (1988). We find the Balmer lines in emission. The spectrum published by Downes \& Keyes (1988) indicates the presence of [NII] and [SII] lines, suggesting the presence of a nebula. We find its spectral type to be B1Iape. High galactic latitude, far-IR colours similar to PNe, an early supergiant spectrum with Balmer, $[\mathrm{NII}]$ and $[\mathrm{SII}]$ lines in emission and the absence of [OIII] $5007 \AA$ suggest that it is a very young and very low excitation planetary nebula.

\# IRAS 19590-1249 (LS IV -12 111)

Based on the IRAS data and high galactic latitude, Parthasarathy $(1990,1993 \mathrm{a})$ classified it as a hot postAGB star. Analysis of the high resolution spectrum by Conlon et al. (1993) and Garcia-Lario et al. (1997) suggests that it is a hot post-AGB star evolving into a low excitation planetary nebula.

\section{Conclusions}

From an analysis of the low resolution spectra of several IRAS sources with far-IR colours similar to PNe, several hot post-AGB candidates are indentified.

Acknowledgements. This research was supported in part by grants from the Space Telescope Science Institute (GO-05858) and the National Science Foundation (AST-9819835).

\section{References}

Acker A., Ochsenbein F., Stenholm B., Tylenda R., Marcout J., Schohn C., 1992, Strasbourg-ESO Catalogue of Galactic Planetary Nebulae 
Allen D.A., 1978, MNRAS 184, 601

Allen D.A., 1982, in IAU Colloq. 70 On the Nature of Symbiotic Stars, Friedjung M. and Viotti R. (eds.). Reidel, p. 225

Arkhipova V.P., Ikonnikova N.P., Noskova R.I., Sokol G.V., Esipov V.F., Klochkova V.G., 1999, Astron. Lett. 25, 25

Bobrowsky M., Zijlstra A.A., Grebel E.K., Tinney C.G., te Lintel Hekkert P., Van de Steene G.C., Likkel L., Bedding T.R., 1995, ApJ 446, L89

Borkowski K.J., Blondin J.M., Harrington J.P., 1997, ApJ 482, L97

Bujarrabal V., Bachiller R., 1991, A\&A 242, 247

Conlon E.S., Dufton P.L., McCausland R.J.H., Keenan F.P., 1993, ApJ 408, 593

De Winter D., The P.S., 1990, Astrophys. Space Sci. 166, 99

Downes R.A., Keyes C.H., 1988, AJ 96, 777

Drilling J.S., 1994, in the MK Process at 50 years, ASP Conf. Ser. 60,57

Drilling J.S., Bergeron L.E., 1995, PASP 107, 846

Garcia-Lario P., Manchado A., Pych W., Pottasch S.R., 1997, A\&AS 126,479

Garcia-Lario P., Riera A., Manchado A., 1998, A\&A 334, 1007

Garcia-Lario P., Riera A., Manchado A., 1999, ApJ 526, 854

Gutierrez-Moreno A., Moreno H., 1998, PASP 110, 458

Hardrop J., Rohlfs K., Slettebak A., Stock J., 1959, Luminous Stars in the Northern Milky-Way. Hamburg, Bergedorf

Henize K.G., 1976, ApJS 30, 491

Hrivnak B.J., Kwok S., Volk K.M., 1989, ApJ 346, 265

Jcoby G.H., Hunter D.A., Christian C.A., 1984, ApJS 56, 257

Kozok J.R., 1985a, A\&AS 61, 387

Kozok J.R., 1985b, A\&AS 62, 7

Kwok S., 1993, ARA\&A 31, 63

Loup C., Forveille T., Nyman L.A., Omont A., 1990, A\&A 227, L29

Parthasarathy M., 1990, IAU Symp. 145, Contributed Papers, Michaud G. (ed.), p. 119
Parthasarathy M., 1993a, in Luminous High-Latitude Stars, ASP Conf. Ser. 45, 173

Parthasarathy M., 1993b, ApJ 414, L109

Parthasarathy M., 1994, in the MK Process at 50 years, ASP Conf. Ser. 60, 261

Parthasarathy M., Garcia-Lario P., Pottasch S.R., Manchado A., Clavel J., de Martino D., Van de Steene G.C.M., 1993, A\&A 267, L19

Parthasarathy M., Garcia-Lario P., Manchado A., Cardoba S.F., 1999, A\&A (submitted)

Parthasarathy M., Pottasch S.R., 1986, A\&A 154, L16

Parthasarathy M., Pottasch S.R., 1989, A\&A 225, 521

Pottasch S.R., Bignell C., Olling R., Zijlstra A.A., 1988, A\&A 205, 248

Pottasch S.R., Parthasarathy M., 1988, A\&A 192, 182

Riera A., Garcia-Lario P., Manchado A., Pottasch S.R., Raga A.C., 1995, A\&A 302, 137

Sahai R., Bujarrabal V., Zijlstra A., 1999, ApJ 518, L115

Sanduleak N., Stephenson C.B., 1973, ApJ 185, 899

Stephenson C.B., Sanduleak N., 1971, Luminous Stars in the Southern Milky Way, Publ. Warner Swasey Obs. 1, 1

Van der Veen W.E.C.J., Habing H.J., 1988, A\&A 194, 125

Vijapurkar J., Drilling J.S., Parthasarathy M., 1997, AJ 114, 1573

Vijapurkar J., Parthasarathy M., Drilling J.S., 1998, Bull. Astron. Soc. India 26, 487

Venn K.A., Smartt S.J., Lennon D.J., Dufton P.L., 1998, A\&A 334,981

Volk K.M., Kwok S., 1989, ApJ 342, 345

Wackerling L.R., 1970, Mem. Roy. Astron. Soc. 73, 153

Walborn N.R., Fitzpatrick E.L., 1990, PASP 102, 379

Yamashita Y., Nariai K., Norimoto Y., 1978, An Atlas of Representative Stellar Spectra. University of Tokyo Press, Tokyo 